These SMEs are mostly run by private enterprises in unorganised sector to avoid legal obligations of Occupational Safety and Health $(\mathrm{OSH})$ provisions. Basic OSH provisions are not maintained in such establishments. This study was conducted in Delhi city of India for the accidents took place between 2011 to 2014 . The objective of the study is to analyse the type of accidents and to establish a relationship between training/experience of workers to occurrence of accidents. At the end of the study it is aimed to design a Participatory Action Oriented Training (PAOT) programme.

Methods Survey and Data analysis methods, followed by applying simple statistical techniques were used for this study. The accident related data for the study period, collected from the labour department and Police stations, were analysed for the study. The study was completed in April-2015

Results It was found that most of the accidents took place in SMEs situated in unorganised/Non industrial areas in Delhi. The causes of accidents are predominantly unsafe machinery or working conditions and lack of training of worker.

Conclusions Adequate training to workers and managing safe and healthy workplace are fundamental responsibility of every employer for prevention of accidents. There is no safety culture in SMEs in India. It has been revealed from this study that two critical points for ultimate results of accidents in SMEs are: unsafe machinery/equipment or working condition and lack of workers training and experience. High emphasis needs to be given to develop safe workplace and workers training. This is possible in large extent by Participatory Action Oriented Training (PAOT), easily available and low cost solutions.

\section{THE INFLUENCE OF PARENTS AND PEERS ON ADOLESCENT ATTITUDES TOWARD HELMET USE IN THE UNITED STATES}

Caroline I McNicholas. Towson University, USA

\subsection{6/injuryprev-2016-042156.1006}

Background Bicycling is a common form of recreation and physical activity for adolescents. The World Health Organisation (WHO) recommends the use of helmets to decrease the chance of injury during wheeled activities. Despite these recommendations, helmet-use rates in the United States are low (i.e., 5\%20\%). One reason may be that adolescents fear that helmet-use may be viewed negatively by peers. The purpose of the study was to examine adolescent attitudes toward helmet use among hypothetical peers and determine whether parent and peer helmet-use influenced adolescents' decisions to wear helmets.

Methods Participants were 40 males aged 10-14 $(M=12.03$, $S D=1.48)$ from a school in the Midwestern United States. The sample included African American (40\%), Caucasian (30\%), Biracial (18\%), and Hispanic (5\%) youth. All youth reported riding bicycles on a weekly basis. Youth were presented with a series of photographs of same-age, same-sex peers riding bicycles with or without helmets. Perceptions of the hypothetical peer were measured using the Revised Adjective Checklist.

Results The majority of youth (60\%) reported "never" or "rarely" wearing a helmet. Participants selected significantly more positive adjectives (e.g., smart, healthy, honest) to describe the helmeted hypothetical peer $(M=27.32 . S D=3.78)$ than the peer without a helmet $(M=24.66, S D=5.75), t(39)=2.60$, $p=.01$; moderate effect size $d=0.55$. Youth's self-reported helmet-use was significantly higher when their parents required helmet-use, $r(38)=0.63, \mathrm{p}<0.01$, and when friends wore helmets, $r(38)=0.65, p<0.01$.

Conclusions Youth in this study were more likely to wear helmets when friends wore helmets and when parents required helmet use. Contrary to expectations, participants in this study viewed a hypothetical peer wearing a helmet more favourably than a peer who was not wearing a helmet. Future research should examine the disconnect between adolescents' favourable perceptions of helmet-use and their low rates of use.

\section{MONITORING REGIONAL ACCIDENTAL INJURY SITUATION IN FINLAND}

Markus Grönfors, Sakari Kääriäinen, Kati Tiirikainen, Persephone Doupi, Satu Pajala, Anne Lounamaa. National Institute for Health and Welfare (THL), Finland

\subsection{6/injuryprev-2016-042156.1007}

Background Official Statistics of Finland (OSF) are a wide collection of statistics describing society's development and state and must have common quality criteria, which are compatible with European Statistical System quality criteria. However, there is no OSF statistics focused solely on injuries, nor a database on the subject.

The National Institute for Health and Welfare (THL) produces information on Finland's injury statistics. For monitoring regional injury situation, THL produces national injury reports, published since 2009, divided into rescue regions and health care districts. National injury reports are released once a year and are intended to improve regional and local safety planning.

Methods Data is collected from the THL Injury Database which contains all deaths and hospital care periods for injuries and external causes of mortality from 1996 to 2013 (ICD-10 codes S00-T983 and V00-Y98). THL Injury Database's data originates from Finland's National Hospital Discharge register and Statistics Finland's Causes of Death register.

Standardised mortality/morbidity rate based on indirect ageand gender standardisation is used for comparing regional accidental injury deaths, hospital care periods, number of patients and hospital care days.

Results Injuries leading to hospital care periods happens more often in Northern and Eastern Finland than in Southern and Western Finland. Most injuries occur in leisure and free time. Falls are the most common cause of death and reason for hospital care periods in the whole country.

Regional differences are explained among other things by varying alcohol consumption, local care practices and population structure.

Conclusions Regional national injury reports provide up-to-date information on municipalities' injury situation. Injury reports guide local officials involved in safety planning to the right direction.

\section{WHAT FACTORS PREDICT SAFETY BEING VALUED AT WORK?}

Ratilainen Henriikka, Kalakoski Virpi, Ukkonen Antti, Perttula Pia, Puro Vuokko, Salminen Simo, Räsänen Tuula. Finnish Institute of Occupational Health, Finland

10.1136/injuryprev-2016-042156.1008

Background Interest is growing in safety as a value in business life. However, there is no generally accepted definition of safety 